Kompass

Pneumologie

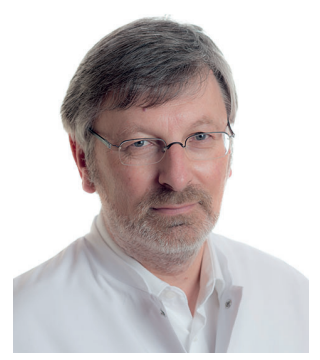

Peter Haidl

Fachkrankenhaus Kloster Grafschaft GmbH, Abteilung Pneumologie II, Schmallenberg, Deutschland

\title{
Pneumologie präzisieren und personalisieren: Perspektiven und Grenzen
}

Liebe Kolleginnen und Kollegen, liebe Leserinnen und Leser,

Sie sehen dem 61. Kongress der Deutschen Gesellschaft für Pneumologie und Beatmungsmedizin (DGP) in Leipzig erwartungsfroh entgegen. Das Kongressthema lautet «Pneumologie - persönlich und präzise», im Programm wird sogar der Begriff der «personalisierten Präzisionsmedizin» verwendet, vielleicht ein weißer Schimmel der modernen Medizin?

Das amerikanische Forschungsprogramm des National Institute of Health (NIH) «Precision Medicine Initiative Cohort Programm» hat diesen Begriff stark ins Bewusstsein gerückt. Ziel soll sein, die Fähigkeit zu verbessern, Erkrankungen vorzubeugen und zu therapieren. Dieser Fortschritt basiert auf der Erkennung genetischer Unterschiede sowie der Berücksichtigung der Lebensweise und der Umweltbedingungen. Wichtig wird dabei sein, diese Fortschritte auch für jedermann zugänglich zu machen. Dies soll genauso für medizinisch unterversorgte und sozial benachteiligte Menschen und Populationen gelten, die in der biomedizinischen Forschung unterrepräsentiert sind. Der Begriff der «Präzisionsmedizin» hat damit sowohl wissenschaftliche als auch gesundheits- und gesellschaftspolitische Aspekte. In diesem Zusammenhang hat der
Kongress die Aufgabe, Wissen und Fortschritte so zu vermitteln, dass diese nicht nur einer kleinen Gruppe von Spezialisten zur Verfügung stehen, sondern auch in der Basisversorgung ankommen.

In der Therapie bedeutet dies die Abkehr von einer «Mittelwert-

Medizin» zu einem vermehrten Augenmerk auf die Besonderheiten der Responder.

Das klassische differenzialdiagnostische Herangehen an den Patienten mit «signs und symptoms» [1] wird nicht an Bedeutung verlieren. Die sorgfältige Anamnese und Untersuchung des Patienten wird die Basis der nachfolgenden diagnostischen und therapeutischen Schritte bleiben. Eine Vielzahl zusätzlicher Daten (Genetik, Biomarker, Bildgebung, Funktionsdiagnostik) helfen bereits jetzt und gewiss auch verstärkt in der Zukunft, Patientenkollektive (Cluster) präziser zu definieren. In der Therapie bedeutet dies die Abkehr von einer «Mittelwert-Medizin» zu einem vermehrten Augenmerk auf die Besonderheiten der Responder.

Eine wichtige Frage wird sein, wie die Fülle an Daten zusammengeführt wird, um daraus therapeutische Interventionen abzuleiten. Ein Übermaß an Daten kann aber dazu information@karger.com

() 2020 S. Karger GmbH, Freiburg

www.karger.com/kkp

Karger ${ }^{\prime}=$
Dr. Peter Haidl

Fachkrankenhaus Kloster Grafschaft GmbH

Abteilung Pneumologie II

Annostraße 1, 57392 Schmallenberg, Deutschland

p.haidl@fkkg.de 
führen, dass relevante Zusammenhänge maskiert werden. Die Herausforderung wird deshalb sein, die wirklich relevanten Daten zu identifizieren.

Aktuell hat es den Anschein, dass die Komplexität eher auf einen einzigen Biomarker (Eosinophile bei Asthma, einzelne Tumormutationen bei Lungenkrebs) reduziert wird. Bei der Lungenfibrose wird primär das Computertomogramm verwendet, um z.B. die Subgruppe der an idiopathischer pulmonaler Fibrose (IPF) Erkrankten zu identifizieren, die dann von einer antifibrotischen Therapie profitiert. Mit einer Indikationserweiterung der Substanzen auf die «progrediente fibrosierende interstitielle Lungenerkrankung (ILD)» wird die Patientenzielgruppe deutlich größer [2]. Möglicherweise wird dadurch die Tiefe der Diagnostik wieder abnehmen, d.h. diese wird durch die therapeutischen Möglichkeiten verändert.

Präzisionsmedizin wird ein komplexer, fortlaufender Prozess sein und bleiben, der valide Rückkoppelungs-Mechanismen benötigt [3]. Es werden neue Diagnosemöglichkeiten entstehen, die durch automatisierte Systeme (z.B. auf der Basis von Machine Learning - insbesondere Deep-LearningAlgorithmen) zugeordnet werden. Die Hoffnung ist daher groß, dass neu entwickelte Therapien für Patienten sicherer und wirksamer sein werden.

Dem Kongressmotto möchten wir mit den Beiträgen in diesem ersten Heft des neuen Jahrgangs unserer Zeitschrift KARGER KOMPASS PNEUMOLOGIE Rechnung tragen.
Ich wünsche Ihnen eine anregende Lektüre und einen gewinnbringenden und erlebnisreichen Kongress

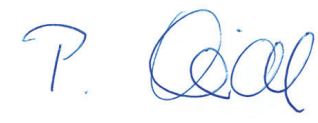

Ihr

Dr. Peter Haidl

\section{Literatur}

1 Novelli, G: Personalized genomic medicine. Int Emerg Med 2010;5(Suppl 1):S81-90.

2 Flaherty KR, Wells AU, Cottin V, et al.: Nintedanib in progressive fibrosing interstitial lung disease. N Eng J Med 2019;381:1718-1727.

$\checkmark 3$ König IR, Fuchs O, Hansen G, et al.: What is precision medicine? ERJ 2017;50(4):pii:1700391. 